\title{
Largest Lyapunov Exponent for Many Particle Systems at Low Densities
}

\author{
R. van Zon, ${ }^{1}$ H. van Beijeren, ${ }^{1}$ and Ch. Dellago ${ }^{2}$ \\ ${ }^{1}$ Institute for Theoretical Physics, University of Utrecht,Postbus 80006, 3508 TA, Utrecht, The Netherlands \\ ${ }^{2}$ Department of Chemistry, University of California, Berkeley, California 94720
}

(Received 27 October 1997)

\begin{abstract}
The largest Lyapunov exponent $\lambda^{+}$for a dilute gas with short range interactions in equilibrium is studied by a mapping to a clock model, in which every particle carries a watch, with a discrete time that is advanced at collisions. This model has a propagating front solution with a speed that determines $\lambda^{+}$, for which we find a density dependence as predicted by Krylov, but with a larger prefactor. Simulations for the clock model and for hard sphere and hard disk systems confirm these results and are in excellent mutual agreement. They show a slow convergence of $\lambda^{+}$with increasing particle number, in good agreement with a prediction by Brunet and Derrida. [S0031-9007(98)05472-6]
\end{abstract}

PACS numbers: 05.20.Dd, 03.20.+i, 05.45.+b,

Recently, there has been great interest in the relationship between statistical mechanics and the theory of dynamical systems [1-3]. Calculating dynamical properties such as Lyapunov exponents for statistical mechanical systems usually requires numerical simulations. For the Lorentz gas, however, Dorfman, Van Beijeren, and others $[3,4]$ have obtained analytical expressions for the Lyapunov spectrum and Kolmogorov-Sinai entropy at low densities, both in equilibrium and for the field-driven case.

In this paper we present an analytic calculation of the largest Lyapunov exponent in the low density limit for a gas at equilibrium consisting of particles with short range interactions. Our method is based on arguments from kinetic theory and similar in spirit to the method of Refs. [3,4]. We compare our results to those from computer simulations on hard disk and hard sphere systems and pay special attention to the dependence of the largest Lyapunov exponent on the total number of particles.

We consider a gas consisting of $N$ atoms of diameter $\sigma$, defined as the (strictly finite) range of interaction, and mass $m$ in $d$ dimensions, in a volume $V$. The reduced density $\tilde{n}$ is defined as $N \sigma^{d} / V$ and will serve as a small parameter. To calculate the largest Lyapunov exponent we follow two nearby trajectories in phase space. For the first one, the reference trajectory, the positions and velocities of the particles are denoted by $\left(\vec{r}_{i}, \vec{v}_{i}\right)$. In the second trajectory they are denoted by $\left(\vec{r}_{i}+\delta \vec{r}_{i}, \vec{v}_{i}+\delta \vec{v}_{i}\right)$. The deviations $\left(\delta \vec{r}_{i}, \delta \vec{v}_{i}\right)$ will be taken to be infinitesimally small. For a chaotic system, they will grow exponentially with time at a rate equal to the largest Lyapunov exponent $\lambda^{+}$. Since the whole vector $\left(\delta \vec{r}_{i}, \delta \vec{v}_{i}\right)$ in phase space grows exponentially, so will a generic projection; hence, one has

$$
\lambda^{+}=\lim _{t \rightarrow \infty} \frac{1}{2 t} \ln \left[\frac{\sum_{i=1}^{N}\left\|\delta \vec{v}_{i}(t)\right\|^{2}}{\sum_{i=1}^{N}\left\|\delta \vec{v}_{i}(0)\right\|^{2}}\right] .
$$

Therefore, in order to calculate $\lambda^{+}$, one has to find out how $\delta \vec{v}_{i}(t)$ typically increases with time. We will first illustrate this on the somewhat simpler case of the random Lorentz gas, consisting of a single light particle moving among a random array of fixed scatterers interacting with the light particle through a spherically symmetric potential. Between collisions the velocity deviation does not change and the position deviation changes according to

$$
\delta \vec{r}(t)=\delta \vec{r}\left(t_{0}\right)+\left(t-t_{0}\right) \delta \vec{v}\left(t_{0}\right) .
$$

In a collision the velocity changes from $\vec{v}$ to $\vec{v}^{\prime}$ given by

$$
\vec{v}^{\prime}=\vec{v}-2(\hat{n} \cdot \vec{v}) \hat{n} \equiv \mathbf{M}_{\hat{n}} \vec{v} .
$$

$\hat{n}$ denotes the unit vector in the direction from the center of the scatterer to the point of closest approach. The change of $\delta \vec{v}$ in a collision is obtained from Eq. (3) by expanding both $\vec{v}+\delta \vec{v}$ and $\hat{n}+\delta \hat{n}$ to linear order in the deviations. The difference in impact times for the two nearby trajectories leads to a shift in $\delta \vec{r}$. Since deviations follow linearized dynamics, one always has

$$
\left(\begin{array}{c}
\delta \vec{r}^{\prime} \\
\delta \vec{v}^{\prime}
\end{array}\right)=\left(\begin{array}{cc}
\mathbf{A} & 2 \mathbf{P} \\
-2 \mathbf{Q} & \mathbf{B}
\end{array}\right)\left(\begin{array}{c}
\delta \vec{r} \\
\delta \vec{v}
\end{array}\right) .
$$

For hard sphere scatterers with radius $\sigma$ it turns out that, in any number of dimensions, $\mathbf{A}=\mathbf{B}=\mathbf{M}_{\hat{n}}, \mathbf{P}=0$, and

$$
\mathbf{Q}=[\sigma(\hat{n} \cdot \vec{v})]^{-1}[(\hat{n} \cdot \vec{v}) \mathbf{1}+\hat{n} \vec{v}] \cdot[(\hat{n} \cdot \vec{v}) \mathbf{1}-\vec{v} \hat{n}],
$$

with 1 the identity matrix. A derivation of these results in two dimensions can be found in [5]. From the above equations we infer that at low density, just after the $k$ th collision, with $k$ very large, $\delta \vec{v}$ and $\delta \vec{r}$ will typically have increased to

$$
\delta \vec{v}^{\prime}\left(t_{k}\right) \approx v(\alpha / \tilde{n})^{k}, \quad \delta \vec{r}^{\prime}\left(t_{k}\right) \approx \sigma(\alpha / \tilde{n})^{k},
$$

with $v$ the speed of the light particle and $\alpha$ a constant of order unity. This follows from an inductive argument: Suppose Eq. (6) is valid after the $k$ th collision, then according to Eqs. (2) and (6) one has $\delta \vec{r}\left(t_{k+1}\right) \approx$ $\delta \vec{r}^{\prime}\left(t_{k}\right)+t_{m f} \delta \vec{v}^{\prime}\left(t_{k}\right) \approx \sigma(\alpha / \tilde{n})^{k+1}$, where we replaced 
$t_{k+1}-t_{k}$ by its average value, the mean free time $t_{m f}$. In the last approximate equality we neglected $\delta \vec{r}^{\prime}\left(t_{k}\right)$ since it is one order of $\tilde{n}$ smaller than $t_{m f} \delta \vec{v}^{\prime}\left(t_{k}\right)$. According to Eqs. (4) and (6), after the $(k+1)$ th collision $\delta \vec{v}^{\prime}\left(t_{k+1}\right)=\delta \vec{v}\left(t_{k}\right)-2 \mathbf{Q} \delta \vec{r}\left(t_{k+1}\right) \approx v(\alpha / \tilde{n})^{k+1}$, where we neglected $\delta \vec{v}\left(t_{k}\right)$ since it is one order of $\tilde{n}$ smaller than the second term and used the typical size of the matrix elements of $\mathbf{Q}$ as $(v / \sigma)$, as is seen explicitly in Eq. (5). Now, because $t \approx k t_{m f} \equiv k / \nu$, with $\nu$ the single particle collision frequency, it follows from Eqs. (1) and (6) that the Lyapunov exponent is

$$
\lambda^{+}=-\nu \ln \tilde{n}+\nu \ln \alpha,
$$

with $\alpha$ to be determined by an averaging procedure over free flight times and collision dynamics. This estimate was already obtained by Krylov [6]. Notice that the value of $\alpha$ is not important for the dominant first term in $\lambda^{+}$.

These considerations can be generalized to systems of identical moving particles by noting that in a collision, say, between particles 1 and 2, Eqs. (3)-(5) are still applicable to the relative velocity $\vec{v}=\vec{v}_{1}-\vec{v}_{2}$, the relative velocity deviation $\delta \vec{v}=\delta \vec{v}_{1}-\delta \vec{v}_{2}$, and the relative position deviation $\delta \vec{r}=\delta \vec{r}_{1}-\delta \vec{r}_{2}$. In addition, one needs the corresponding relations for the center of mass coordinates $\vec{V}=\left(\vec{v}_{1}+\vec{v}_{2}\right) / 2$ and $\vec{R}=\left(\vec{r}_{1}+\right.$ $\left.\vec{r}_{2}\right) / 2$, which are

$$
\vec{V}^{\prime}=\vec{V}, \quad \delta \vec{V}^{\prime}=\delta \vec{V}, \quad \delta \vec{R}^{\prime}=\delta \vec{R} .
$$

Assume now that the deviations for particles 1 and 2, just after their last collisions before the present one, were of the form (6) with exponents $k_{1}$ and $k_{2}$, respectively, and with $v$ the mean relative velocity. By a reasoning similar to that for the Lorentz gas, it follows that just before collision $\delta \vec{v}$ and $\delta \vec{V}$ are both of order $(\alpha / \tilde{n})^{\max \left(k_{1}, k_{2}\right)}$, whereas $\delta \vec{r}$ and $\delta \vec{R}$ are of order $(\alpha / \tilde{n})^{\max \left(k_{1}, k_{2}\right)+1}$. As a consequence of (4) and (7), right after the collision, $\delta \vec{r}_{i}^{\prime}$ and $\delta \vec{v}_{i}^{\prime}$ $(i=1,2)$ will then also be of order $(\alpha / \tilde{n})^{\max \left(k_{1}, k_{2}\right)+1}$. So, on average, $\ln \left|\delta \vec{v}_{i}\right|$ also increases by units of $\ln (\alpha / \tilde{n})$ at collisions, but in contrast to the Lorentz gas this increase may involve several of these units, in case the other particle involved in the collision has a higher $k$ value.

The values of $\ln \alpha$ in an actual realization of the dynamics will fluctuate strongly from collision to collision. However, their distribution becomes independent of density and increasingly narrow relative to $\ln (1 / \tilde{n})$ as density gets closer to zero. Therefore the essence of the dynamics determining the largest Lyapunov exponent is captured in the following simple clock model: Think of each particle $i$ as carrying a watch, whose clock value is $k_{i}$. When two particles collide, they synchronize their watches to the larger of the two clock values, and advance them by one unit. The largest Lyapunov exponent will be determined by the speed $w$ by which the watches run, on average, and will be of the form

$$
\lambda^{+}=w(-\nu \ln \tilde{n}+\nu \ln \alpha) .
$$

The synchronization of the $k$ values prohibits a direct identification with the number of collisions which we could do in the Lorentz gas.

We will use a mean field approach to calculate the clock speed $w$. We denote the number of particles that have a given clock value $k$ by $N_{k}$ and assume that they are distributed uniformly in $V$. In collisions involving particles with clock value $k, N_{k}$ decreases. It is increased by two in collisions in which the largest incoming $k_{i}$ was $k-1$. So the rate equations for the $N_{k}$ become

$$
\frac{d N_{k}}{d t}=-\sum_{\substack{l=-\infty \\ l \neq k}}^{\infty} R_{(k, l)}-2 R_{(k, k)}+2 \sum_{l=-\infty}^{k-1} R_{(k-1, l)} .
$$

$R_{(k, l)}$ are the rates by which collisions between $k$ and $l$ take place. We use a Stoßzahlansatz: The rate of collisions between particles with clock values $k$ and $l$ is proportional to $N_{k} N_{l} / N^{2}$. Since all rates are also proportional to $\nu$, we will express time in units of the mean free time, $\tau=\nu t$. We use the fractions $f_{k}=$ $N_{k} / N$ to eliminate the $N$ dependence:

$$
\frac{d f_{k}}{d \tau}=-f_{k}+2 f_{k-1} \sum_{l=-\infty}^{k-2} f_{l}+f_{k-1}^{2} .
$$

For the cumulatives $C_{k}=\sum_{i=-\infty}^{k} f_{i}$, this reduces to

$$
\frac{d C_{k}}{d \tau}+C_{k}=C_{k-1}^{2} .
$$

The solution is given by the recursion relation

$$
C_{k}(\tau)=e^{-\tau} C_{k}(0)+\int_{0}^{\tau} e^{\tau^{\prime}-\tau} C_{k-1}^{2}\left(\tau^{\prime}\right) d \tau^{\prime} .
$$

If $C_{k}$ is zero at $\tau=0$ it remains zero. Thus the starting point of this recursion is the smallest $k$ for which $C_{k}(0) \neq$ 0 . Inductively, we see that all $C_{k}$ are polynomials in $e^{-\tau}$, of which the degree grows exponentially with $k$. We calculated these polynomials with initial conditions corresponding to $f_{k}(\tau=0)=\delta_{k 1}$. The exponentially growing degree of the polynomials enables only a limited number of $C_{k}$ to be computed, even on a computer. The results up to $k=30$ at several time values are shown in Fig. 1. The initial distribution broadens and moves to the right. We expect the distribution to asymptotically become a front propagating at a constant speed $w$. Then we have

$$
\begin{aligned}
\sum_{i=1}^{N}\left\|\delta \vec{v}_{i}(t)\right\|^{2} & =\sum_{k=-\infty}^{\infty} f_{k}(\tau) v^{2} e^{-2 k \ln (\tilde{n} / \alpha)} \\
& \simeq \sum_{k=-\infty}^{\infty} f_{k}(0) v^{2} e^{-2(k-w \tau) \ln (\tilde{n} / \alpha)}
\end{aligned}
$$

This result should be proportional to $e^{2 t \lambda^{+}}$so one indeed recovers Eq. (8). It agrees with Krylov's conjecture [6], except for the appearance of $w$. Several years ago 


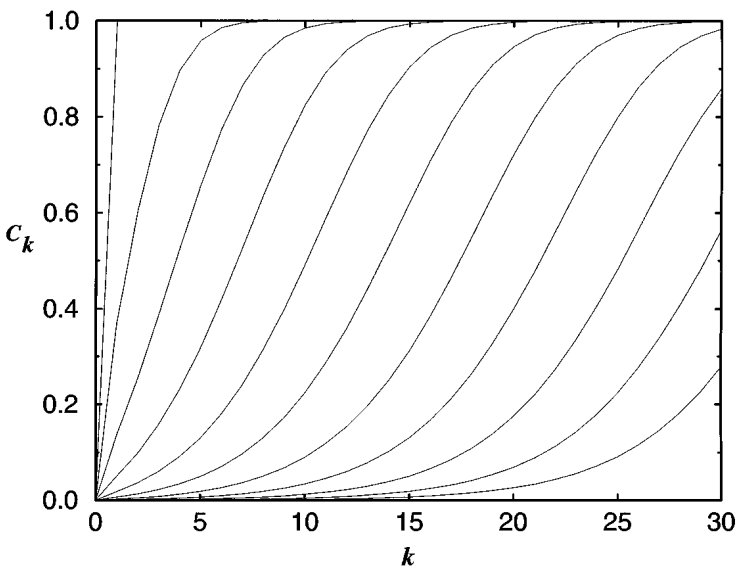

FIG. 1. The cumulative distribution of clock values $k$ at times $\tau=0, \tau=1, \tau=2, \ldots, \tau=10$ (from left to right).

Stoddard and Ford [7] obtained results that are of the same form when expanded in $\tilde{n}$.

The speed $w$ should be independent of the initial conditions. Physical initial conditions will have finite support, because there is always a particle with smallest $k$ and a particle with highest $k$. Then $C_{k}(0)$ is bounded by two step functions. Using Eq. (9), one can show that they will remain bounded by the solutions corresponding to these two initial conditions. If these tend to some uniformly moving solution with speed $w$, so will the real system. Thus $w$ is unique for this set of initial conditions.

We put the propagating front ansatz $C_{k}(\tau)=F(k-$ $w \tau)$ into Eq. (9) to obtain a differential-difference equation [8] for the shape of the cumulatives:

$$
-w \frac{d F}{d x}(x)+F(x)=F^{2}(x-1) .
$$

$F$ has to be monotonically increasing, tending to 0 as $x \rightarrow-\infty$ and to 1 as $x \rightarrow \infty$. This means that $F=0$ has to be unstable and $F=1$ has to be stable. It is easy to see that these are fixed points of Eq. (10). Their stability is determined by linearized equations. The behavior around a fixed point is always exponential: $F(x)=\sum_{j} p_{j} e^{s_{j} x}$, in which the $s_{j}$ are roots of the so-called characteristic equation and $p_{j}$ are polynomials in $x$ of a degree less than the multiplicity of root $s_{j}$ [8]. For an unstable fixed point, some of the $s_{j}$ have positive real parts. Around $F=0$, this is true if $w>0$. For a stable fixed point, the term with least negative $s_{j}$, lets call this $-\gamma$, will dominate the large $x$ behavior. If $\gamma$ were complex, we would see oscillatory behavior, violating monotonicity, so $\gamma$ has to be real. Inserting the asymptotic behavior $F(x)=1-$ $\exp (-\gamma x)$ into Eq. (10) and neglecting quadratic terms produce the characteristic equation $\gamma w+1-2 e^{\gamma}=0$. This gives a relation between $w$ and $\gamma: w(\gamma)=\left(2 e^{\gamma}-\right.$ $1) / \gamma$. It turns out that there is a minimum $w$ for positive real values of $\gamma$ which can be expressed in terms of Lambert's $W$ function

$$
w=-1 / W(-1 / 2 e)=4.31107 \ldots
$$

This value is in accordance with estimates from Fig. 1. Solutions with initial conditions with finite support select this minimum speed. The same type of velocity selection occurs in other systems, a number of which have been proved [9].

We compared the result $w=4.311 \ldots$ with those from simulations done by Dellago, Posch, and Hoover [5], with 64 hard disks. They made a fit of the largest Lyapunov exponent to $a n \ln (n / b)$ indicating a value of $w \approx 3.3$. The difference to our value turns out to be due to large finite $N$ effects. We will first show this numerically. We take $N$ watches and give them some initial $k$ values. In each time step we pick two watches at random and advance their $k$ values according to the rules of the clock model. We compute the average growth of $k$ per watch per time step, and find $w_{N}$. We did this for a number of watches ranging from 4 to $2^{19}$. In Fig. 2 the results were fitted to an algebraic curve,

$$
w_{N}=4.311-A N^{-B} .
$$

A good fit, except for the smallest values of $N$, was obtained by choosing $B=0.277$ and $A=3.466$. No good fit could be obtained for $B=1$ or on replacing the algebraic $N$ dependence by an exponential one. The value $B=0.277$ is in reasonable agreement with the exponent of $-1 / 3$ obtained by Dellago and Posch [10]. In order to obtain a better comparison between clock model predictions for $w_{N}$ and simulation results on actual dilute gas models, we performed a number of new simulations using the same methods as in Refs. [5,10], both on hard disk and hard sphere systems for different particle numbers at a number of low densities, $\tilde{n}=10^{-5}, 10^{-4}$,

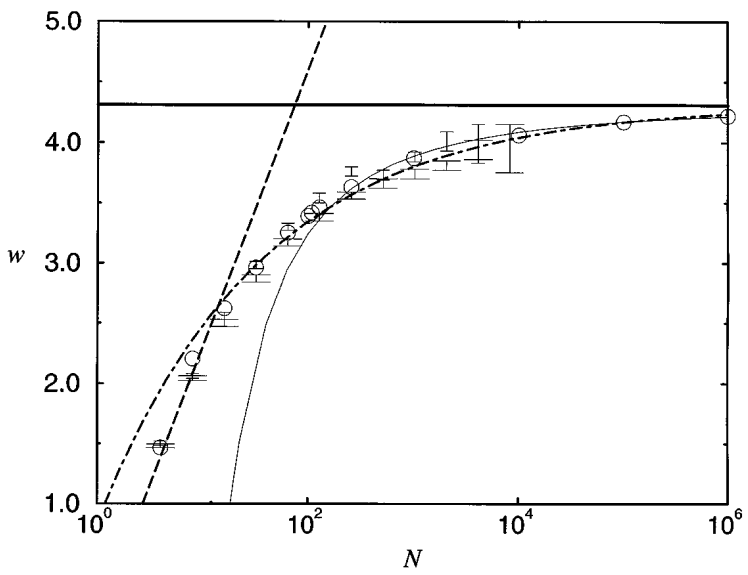

FIG. 2. Number dependent coefficient $w$. The circles are clock model results. The bars are new molecular simulation results: wide error bars for hard spheres and narrow error bars for hard disks. The dashed line is Stoddard and Ford's $\ln N$ prediction. The dash-dotted curve is a fit of the mean field results to the algebraic expression (11). The thick line gives the analytic result for $N \rightarrow \infty$. The solid curve is the prediction of Eq. (12). 
$10^{-3}$, and $10^{-2}$. For each $N$ the results for the largest Lyapunov exponent were fitted to $-w \nu \ln (\tilde{n} / \alpha)$. The results for $w$ are also plotted in Fig. 2. One sees that the results of the clock model are in excellent agreement with those of the simulations.

Stoddard and Ford [7] used crude arguments to get $w_{N}=\ln N$. This relation is also plotted in Fig. 2. One sees that this gives only a good fit for very small $N$. In simulations of 100 particles with a cutoff LennardJones interparticle potential, Stoddard and Ford [7] found agreement with their predicted value, which lies somewhat above our asymptotic value of 4.311 and much more above the simulation results for 100 particles, both in the clock model and for hard spheres and disks. Stoddard and Ford [7] acknowledged that the error increases as $\tilde{n}$ gets smaller and that their simulation results for low $\tilde{n}$ should not be expected to fit their theory. But the $\tilde{n}$ at which the error becomes too big is not sharply defined. If one takes it low enough, the data support their prediction; but if one takes it a little higher, the results are more in line with those from the clock model simulations.

In a recent paper, Brunet and Derrida [11] present a way to compute the $N$ dependence of the velocity in a similar model by treating it as a discretization effect. In our case, there are at least two particles with highest $k$ in any realization. This means we have to take $\epsilon=2 / N$ in Eq. (7) in [11]. Inserting our expression for $w(\gamma)$, we find

$$
w_{N}=w-\frac{(w-1) \pi^{2}}{2 \ln ^{2}(N / 2)}
$$

We also plotted this prediction in Fig. 2. The agreement is good for $N>100$.

In the work by Searles et al. [12] a weak but persistent increase in $\lambda^{+}$with $N$ was interpreted as a sign of a logarithmic divergence. It was argued that the data were not consistent with a $1 / N$ approach to a constant value, and a plot of $\lambda^{+}$versus $\ln N$ looks quite linear over an appreciable range. However, in their simulations on dense hard sphere systems, Dellago and Posch [10] did not observe such a divergence and, in fact, it looks like the results of Searles et al. are entirely consistent with the type of behavior predicted by Brunet and Derrida [11]. The mean field analysis given here is not decisive though, since it completely ignores all effects of local density and temperature fluctuations.

We conclude by stressing that the first term of the density expansion of the largest Lyapunov exponent of a dilute gas that was calculated in this Letter is universal for systems where the interaction is sufficiently short ranged, i.e., it strictly vanishes beyond its range $\sigma$, or perhaps may be allowed to vanish exponentially. This Letter shows that the calculation of dynamical properties of many particle systems is feasible and that the calculation of the largest Lyapunov exponent in dilute gases requires the solution of a nonlinear front propagation equation. The method will be extended in future work in order to obtain the $\mathcal{O}(\tilde{n})$ term of the Lyapunov exponent. This term will depend on the details of the interaction at a collision, and is of considerable physical interest.

We thank Professor J.R. Dorfman and Professor H. A. Posch for valuable discussions, Professor Th. W. Ruijgrok for pointing out useful references, and Professor A. Zangwill for providing us with a copy of the doctoral thesis of Stoddard. The work reported here was supported by FOM, SMC, and by the NWO Priority Program Nonlinear Systems, which are financially supported by the "Nederlandse Organisatie voor Wetenschappelijk Onderzoek (NWO)." H. van Beijeren and R. van Zon acknowledge the hospitality and support of the Institute for Physical Science and Technology at the University of Maryland during the first stages of their research. Ch. Dellago gratefully acknowledges support from the Austrian Fonds zur Förderung der wissenschaftlichen Forschung, Grant No. J01302-PHY.

[1] D. J. Evans and G.P. Morriss, Statistical Mechanics of Nonequilibrium Liquids (Academic, London, 1990).

[2] Special issue on the Proceedings of the Euroconference on The Microscopic Approach to Complexity in NonEquilibrium Molecular Simulations. CECAM at ENS-Lyon, 1996, edited by M. Mareschal, Physica (Amsterdam) 240A (1997).

[3] J. R. Dorfman and H. van Beijeren, in Ref. [2], pp. 12-42.

[4] H. van Beijeren and J. R. Dorfman, Phys. Rev. Lett. 74, 4412 (1995); H. van Beijeren, J.R. Dorfman, E. G. D. Cohen, Ch. Dellago, and H. A. Posch, Phys. Rev. Lett. 77, 1974 (1996); A. Latz, H. van Beijeren, and J. R. Dorfman, Phys. Rev. Lett. 78, 207 (1997).

[5] Ch. Dellago, H. A. Posch, and W. G. Hoover, Phys. Rev. E 53, 1485-1501 (1996).

[6] N.S. Krylov, Works on the Foundations of Statistical Physics (Princeton University Press, Princeton, 1979).

[7] S. D. Stoddard and J. Ford, Phys. Rev. A 8, 1504 (1973).

[8] R.E. Bellman and K.L. Cooke, Differential-Difference Equations (Academic, New York, 1963).

[9] R. D. Benguria and M. C. Depassin, Phys. Rev. Lett. 77, 1171 (1996).

[10] Ch. Dellago and H. A. Posch, in Ref. [2], pp. 68-83.

[11] E. Brunet and B. Derrida, Phys. Rev. E 56, 2597 (1997).

[12] D. J. Searles, D. J. Evans, and D. J. Isbister, in Ref. [2], pp. 96-104. 